\title{
Gravitational major-axis contraction of Mercury's elliptical orbit
}

\author{
Q. H. Liu,,${ }^{1,2, \text { * } \mathrm{Q} . \mathrm{Li},{ }^{1} \text { T. G. Liu, }{ }^{1} \text { and X. Wang }}{ }^{1}$ \\ ${ }^{1}$ School for Theoretical Physics, School of Physics and Electronics, Hunan University, Changsha 410082, China \\ ${ }^{2}$ Synergetic Innovation Center for Quantum Effects and Applications (SICQEA), \\ Hunan Normal University, Changsha 410081, China
}

(Dated: February 18, 2019)

\begin{abstract}
The local curvature of the space produced by the Sun causes not only the perihelion precession of Mercury's elliptical orbit, but also the variations of the whole orbit, in comparison with those predicted by the Newtonian theory of gravitation. Calculations show that the gravitational majoraxis contraction of the Mercury's elliptical orbit is 1.3 kilometers which can be confirmed by the present astronomical distance measurement technology.
\end{abstract}

\section{INTRODUCTION}

Mercury is the planet closest to the Sun, the swiftest in the solar system [1, 2]. She revolves around the Sun in an elliptical orbit at a mean distance of about 57.9 million $\mathrm{km}$; the orbital period is 0.24 Earth years [1, 2]. At perihelion the Mercury is $r_{p} \approx 46.0$ million $\mathrm{km}$ from the Sun, and at aphelion it is at a distance of $r_{a} \approx 69.8$ million $\mathrm{km}$, and the orbital eccentricity $e$ which is rather large $(e \approx 0.2056)[1,2]$ in comparison with that of the Earth $(e \approx 0.0167)$. It was a historical puzzling that there was from the Newtonian theory of gravitation an anomalous precession of the perihelion of Mercury, about 43 seconds of arc per century, and in 1915 Einstein proposed to take such a procession as an observational evidence for the test of his theory of general relativity [4]. Nowadays, it is the standard approach that the gravitation must be understood within the general relativity. A curious question on the gravitational influence on the Mercury's elliptical orbit is in the following. For an elliptical orbit with Sun at one focus, one can conveniently choose the focus as origin of polar coordinates, and there are two coordinates, polar angular and radial ones, usually denoted by $\varphi$ and $r$, respectively, to specify the position of the planet. The gravitation must simultaneously have effects on both the angular and radial motions. We can ascertain that the gravitationally induced change of the radial motion is small, and had been hard to be observed when the general relativity was establishing in 1915 and some years later, and it may be testable today. The present paper is devoted to study the gravitational major-axis contraction of Mercury's elliptical orbit. In the present paper, we consider only the effects of the local curvature of the space produced by the Sun on the motion of the Mercury, without taking other gravitational perturbations coming from, e.g., other planets and oblateness of the Sun etc., into account.

This paper is organized in the following. In section II, the gravitational major-axis contraction of Mercury's elliptical orbit is defined as the difference between two (proper) distances between the perihelion and the aphelion of the Mercury's orbit, predicted by the Einstein's and Newtonian theory of the gravitation, respectively. In section III, the new perihelion $r_{P}$ and aphelion $r_{A}$ of the Mercury's orbit in the Einstein's theory of gravitation are calculated. In final section IV, a brief conclusion is given.

\section{DEFINITIONS OF TWO LENGTHS OF THE MAJOR-AXIS OF THE MERCURY'S ELLIPTICAL ORBIT IN MODERN AND CLASSICAL THEORY OF GRAVITATION}

For an ellipse whose equation is, with $p \equiv a\left(1-e^{2}\right)$ and $a$ standing for the semi-major axis,

$$
r=\frac{p}{1+e \cos \varphi}
$$

the area $S=\pi a^{2} \sqrt{1-e^{2}}$, and the perimeter $C=4 a E(e)$ with $E(e)$ denoting the complete elliptic integral of the second kind. For a circle of radius $a$, we have with $e=0, S=\pi a^{2}$ and $C=4 a E(0)=2 \pi a$ with $E(0)=\pi / 2$.

*Electronic address: quanhuiliu@gmail.com 
In the Newtonian theory of gravitation for planets in the solar system, the elliptical orbit satisfies following Binet's equation, [3]

$$
\frac{d^{2} u}{d \varphi^{2}}+u=b^{2}
$$

where $u=r_{g s} / r$ with $r_{g s} \equiv G M / c^{2}$ denoting the gravitational radius of the Sun, and GM being the product of gravitational constant and the mass of the Sun and $c$ being the velocity of light speed, and $b=G M /(c L)=r_{g s} c / L$ with $L$ being the dimensionless specific angular momentum. For the Sun, $r_{g s} \sim 1.47 \mathrm{~km}$, and for Mercury, mean value of radius is $\bar{r} \sim 57.9$ million $\mathrm{km}$, we have $b^{2} \sim \bar{u}=r_{g s} / \bar{r} \approx 0.25 * 10^{-7}$. The solution to equation (2) is,

$$
u=u_{0} \equiv b^{2}(1+e \cos \varphi) \text {. }
$$

This result is purely a geometric one, given by the Newtonian theory of gravitation. The perihelion $r_{p}$ and aphelion $r_{a}$ are, respectively,

$$
r_{p}=\frac{1}{b^{2}(1+e)} \approx 46.0 \text { million } \mathrm{km}, \text { and } r_{a}=\frac{1}{b^{2}(1-e)} \approx 69.8 \text { million } \mathrm{km} .
$$

However, the astronomical observations indicate that the orbit is not exactly closed as suggested by (3), and the Einstein's theory of gravitation must be utilized to understand the anomalous behavior of the Mercury's orbit.

The Einstein's theory of gravitation presents that the external gravitational field produced by the Sun has spherical symmetry and is usually expressed by the Schwarzschild metric, [3 5 ]

$$
d s^{2}=-\left(1-\frac{2 r_{g s}}{r}\right) d t^{2}+\frac{1}{\left(1-\frac{2 r_{g s}}{r}\right)} d r^{2}+r^{2}\left(d \theta^{2}+\sin ^{2} \theta d \varphi^{2}\right),
$$

where the parameter $s$ denotes the proper length, the parameter $t$ is the coordinate time and $(r, \theta, \varphi)$ are the conventional spherical coordinates. When the Mercury moves in the gravitational field of the Sun, as usual, we choose $\theta=\pi / 2$ as the orbit plane. We can then use the Einstein's theory of gravitation to get the new perihelion $r_{P}$ and the new aphelion $r_{A}$ of the Mercury's orbit. Once these two quantities are known, the proper distance between the perihelion and the aphelion, or the new length of the major-axis $D$, is approximately given by,

$$
D \approx \int_{R_{\odot}}^{r_{P}} \frac{1}{\sqrt{1-\frac{2 r_{g s}}{r}}} d r+\int_{R_{\odot}}^{r_{A}} \frac{1}{\sqrt{1-\frac{2 r_{g s}}{r}}} d r+2 R_{\odot} \approx r_{P}+r_{A}+r_{g s} \ln \frac{r_{P} r_{A}}{R_{\odot}^{2}},
$$

where $R_{\odot} \approx 69.6 * 10^{-2}$ million $\mathrm{km}$ is the mean radius of the Sun. Evidently, the new major-axis $D$ in the Einstein's theory of gravitation comes from two parts,

$$
r_{P}+r_{A} \text { and } r_{g s} \ln \frac{r_{P} r_{A}}{R_{\odot}^{2}} .
$$

The first part $r_{P}+r_{A}$ will be computed in the following section, and turns out to be less than $r_{p}+r_{a}$. The second part proves to be approximately, with $r_{p} \approx 46.0$ and $r_{a} \approx 69.8$ million $\mathrm{km}$ in the Newtonian theory of gravitation,

$$
r_{g s} \ln \frac{r_{P} r_{A}}{R_{\odot}^{2}} \approx r_{g s} \ln \frac{r_{p} r_{a}}{R_{\odot}^{2}} \approx 12.9 \mathrm{~km} .
$$

This mainly results from the curvature of the space induced by the gravitational field of the Sun.

Remember that the Newtonian theory predicts an exactly closed elliptic orbit for the Mercury, but the Einstein's theory correctly gives the precession the perihelion. Though the gravitational length contraction is hard to define in general, the gravitational major-axis contraction of Mercury's elliptical orbit can be well-defined in the similar manner. On one hand, we have the length of the major-axis predicted by the Newtonian theory of gravitation, which is $r_{p}+r_{a}$. On the other, we have that length from the Einstein's theory, which is $D$ (6). Their difference is clearly given by,

$$
D-\left(r_{p}+r_{a}\right)=r_{P}+r_{A}-\left(r_{p}+r_{a}\right)+r_{g s} \ln \frac{r_{P} r_{A}}{R_{\odot}^{2}},
$$

which will be shortly shown to be -1.3 kilometers, which is referred as to the gravitational major-axis contraction of Mercury's elliptical orbit. In following section, we will calculate $r_{P}$ and $r_{A}$. 


\section{NEW PERIHELION $r_{P}$ AND APHELION $r_{A}$ OF THE MERCURY'S ORBIT IN THE EINSTEIN'S THEORY OF GRAVITATION}

Once the gravitation is static and weak, the Einstein's theory of general relativity predicts that Mercury's orbit satisfies following nonlinear equation, an extension of the Binet's equation,

$$
\frac{d^{2} u}{d \varphi^{2}}+u=b^{2}+3 u^{2}
$$

In this equation, the quantity $3 u^{2} \sim 0.19 * 10^{-14}$ is a perturbation in comparison with the first term on the right handed side $b^{2} \sim u \approx 0.25 * 10^{-7}$. This equation is still a geometrical one expressing the relation between angular variable $\varphi$ and the inverse of the dimensionless radial variable $u$. Without the presence of the perturbation, the Binet's equation (2) gives a perfect ellipse, and the effective frequency is unity, as shown in Eq. (3). With the perturbation, one can no longer expect that the frequency remains unity. To solve Eq. (10), we utilize the standard perturbation method [6] to get an approximate solution. For sake of convenience, we introduce a real parameter $\varepsilon$ into equation (10) which then becomes,

$$
\frac{d^{2} u}{d \varphi^{2}}+u=b^{2}+3 \varepsilon u^{2}
$$

which can also be rewritten into following form with a new parameter $\xi \equiv \omega \varphi(\xi \in[0, \infty))$, where $\omega$ differs from unity due to the possibly small frequency renormalization as a consequence of removal of the secular term as shown in (13b),

$$
\omega^{2} \frac{d^{2} u}{d \xi^{2}}+u=b^{2}+3 \varepsilon u^{2} .
$$

In deriving Eq. (10), there is an effective radial confining potential [3 5], from which we know that the particle is bounded between the aphelion and perihelion, and every bound state possesses a fixed frequency. Thus $\omega$ must also be a constant. The perturbation solution to the equation (12) can be expanded in powers of $\varepsilon$, [6]

$$
\begin{aligned}
u & =u_{0}(\xi)+\varepsilon u_{1}(\xi)+\varepsilon^{2} u_{2}(\xi)+\cdots \\
\omega & =1+\varepsilon \omega_{1}+\varepsilon^{2} \omega_{2}+\cdots
\end{aligned}
$$

where the subscripts $0,1,2, \ldots$ in expansions of $u$ and $\omega$ denote the orders of approximation. When all required calculations are complete, we set $\varepsilon=1$, so above expansions are in fact in powers of $u \sim b^{2}$, as it should be.

The zeroth order solution of the elliptical orbit is already given by the Newtonian theory of gravitation, i.e., (3). The first order solution $u_{1}(\varphi)$ satisfies following equation,

$$
\frac{d^{2} u_{1}}{d \xi^{2}}+u_{1}=3 u_{0}^{2}-2 \omega_{1} u_{0}^{\prime \prime}=3 u_{0}^{2}-2 \omega_{1}\left(b^{2}-u_{0}\right)
$$

The initial conditions are, [6]

$$
u_{1}(0)=0, \frac{d u_{1}(0)}{d \xi}=0
$$

The solution to equation (14) is,

$$
u_{1}=2 b^{4}\left(3+2 e^{2}+e^{2} \cos \xi\right) \sin ^{2}(\xi / 2)+b^{2} e\left(\omega_{1}+3 b^{2}\right) \xi \sin \xi .
$$

The second term is secular for it contains $\xi \sin \xi$ which can be infinitely large as $\xi \rightarrow \infty$ and must be eliminated by setting,

$$
\omega_{1}=-3 b^{2}
$$

which accounts for the procession of the perihelion of Mercury. [3 [5] To see it explicitly, we maximize $u \simeq u_{0}(\xi)+$ $u_{1}(\xi)=b^{2}(1+e \cos \xi)+2 b^{4}\left(3+2 e^{2}+e^{2} \cos \xi\right) \sin ^{2}(\xi / 2)$ which is to be $b^{2}(1+e)$ that defines the perihelion of Mercury, when the parameter $\theta$ takes following values,

$$
\xi \equiv \omega \varphi=2 \pi n,(n=0,1,2)
$$


which can be rewritten in terms of the polar angle $\varphi$,

$$
\left(1-3 b^{2}\right) \varphi \approx 2 \pi n
$$

This reproduces the well-known result for the angular difference between two successive points of closest approach of Mercury to the Sun, [3-5]

$$
\Delta \varphi \approx 6 \pi b^{2}
$$

The first order correction of $\varepsilon$ to the inverse of the dimensionless radial position $u=r_{g s} / r$ is positive from (16),

$$
u_{1}=2 b^{4}\left(3+2 e^{2}+e^{2} \cos \xi\right) \sin ^{2}(\xi / 2) \geq 0 .
$$

Combination of this result and the unperturbed one (3) gives,

$$
u \approx b^{2}(1+e \cos \xi)+2 b^{4}\left(3+2 e^{2}+e^{2} \cos \xi\right) \sin ^{2}(\xi / 2)
$$

The radial position is then,

$$
\begin{aligned}
r & \approx r_{g s}\left(b^{2}(1+e \cos \xi)+2 b^{4}\left(3+2 e^{2}+e^{2} \cos \xi\right) \sin ^{2}(\xi / 2)\right)^{-1} \\
& \approx \frac{r_{g s}}{b^{2}(1+e \cos \xi)}\left(1-\frac{2 b^{2}\left(3+2 e^{2}+e^{2} \cos \xi\right) \sin ^{2}(\xi / 2)}{1+e \cos \xi}\right) \\
& \approx r_{g s}\left(\frac{1}{b^{2}(1+e \cos \xi)}-\frac{2\left(3+2 e^{2}+e^{2} \cos \xi\right) \sin ^{2}(\xi / 2)}{(1+e \cos \xi)^{2}}\right)
\end{aligned}
$$

This equation gives both the coordinate of the new perihelion $r_{P}$,

$$
r_{P}=r_{\min }=\left.r\right|_{\xi=0} \approx \frac{r_{g s}}{b^{2}(1+e \cos \xi)}=r_{p}
$$

and the new aphelion $r_{A}$,

$$
r_{A}=r_{\max }=\left.r\right|_{\xi=\pi} \approx \frac{r_{g s}}{b^{2}(1-e)}-r_{g s} \frac{2\left(3+e^{2}\right)}{(1-e)^{2}}=r_{a}-14.2 \mathrm{~km} .
$$

Combining results (24) and (25), we find that the distance between the perihelion and the aphelion, i.e., the length of the major-axis of the ellipse, is shortened, and the difference is,

$$
\Delta r \equiv r_{P}+r_{A}-\left(r_{p}+r_{a}\right)=-r_{g s} \frac{2\left(3+e^{2}\right)}{(1-e)^{2}} \approx-14.2 \mathrm{~km} .
$$

In consequence, we see that result (8) holds true.

Now we are ready to give the gravitational major-axis contraction of the Mercury's elliptical orbit in unit of kilometer,

$$
D-\left(r_{p}+r_{a}\right) \approx-14.2+12.9=-1.3
$$

In a little more detail, in the Newtonian theory of gravitation, we have major-axis $\left(r_{p}+r_{a}\right)$ with the Sun as a point of mass; however, in the Einstein's theory, we have a little bit shorter one $D$, which can be observed with present astronomical distance measurement technology. In the website of NASA, 2] we see that the distance of the Mercury from the Sun is reported in every second with accuracy of one kilometer.

\section{CONCLUSION}

To sum up, since the gravitation between Mercury and the Sun must have influences on both radial and angular positions, we compute the gravitational major-axis contraction of Mercury's elliptical orbit. The result is -1.3 km which can be confirmed in the observation of the Mercury's orbit, 


\section{Acknowledgments}

This work is financially supported by National Natural Science Foundation of China under Grant No. 11675051. We are indebted to an anonymous reviewer for pointing us the proper distance (6), which greatly contributed to improving the final version of the paper.

[1] European Southern Observatory, Mercury's Orbit and Visibility, https://www.eso.org/public/outreach/eduoff/vt-2004/mt2003/mt-mercury-orbit.html.

[2] NASA. Mercury. https://solarsystem.nasa.gov/planets/mercury/overview/, https://solarsystem.nasa.gov/planets/mercury/bythe-numbers/.

[3] C. W. Misner, K. S. Thorne, J. A. Wheeler, Gravitation. (San Francisco: Freeman and Company. 1973).

[4] S. Weinberg, Gravitation and cosmology. (New York: Wiley. 1972).

[5] L. D. Landau, E. M. Lifshitz, The Classical Theory of Fields. Vol. 2 (4th ed.). (Oxford: Butterworth-Heinemann, 1998).

[6] D. R. Smith, Singular-Perturbation Theory, An Introduction with Applications. (Cambridge: Cambridge University Press, 1985) 